\title{
An Unusual Case of Multiple Primary Carcinomas: Breast Cancer and Rectal Adenocarcinoma in a Single Patient: Report of a Case and Review of the Literature
}

\author{
Akasbi $\mathbf{Y}^{1^{\star}}$, Arifi $\mathbf{S}^{1}$, Najib $\mathbf{R}^{1}$, Hammas $\mathbf{N}^{2}$, Amarti $\mathbf{A}^{2}$ and Mellas $\mathbf{N}^{1}$ \\ ${ }^{1}$ Medical Oncology Department, Hassan II University Hospital, Fez, Morocco \\ ${ }^{2}$ Department of Pathology, Hassan II University Hospital, Fez, Morocco \\ *Corresponding author: Akasbi Yousra, Medical Oncology Department, Hassan II University Hospital, Fez, Morocco, Tel : +212664125655; E-mail: \\ you.yous@hotmail.fr
}

Received date: Nov 10, 2015; Accepted date: Dec 26, 2015; Published date: Dec 30, 2015

Copyright: (C) 2015 Akasbi Y, et al. This is an open-access article distributed under the terms of the Creative Commons Attribution License, which permits unrestricted use, distribution, and reproduction in any medium, provided the original author and source are credited.

\begin{abstract}
Introduction: The incidence of multiple primary malignant neoplasms (MPMN) increases with age. While they are nowadays encountered more frequently, the phenomenon is still considered to be rare. Our case represent a rare association between breast and rectal cancer.

Multiplicity of primary malignancies itself does not necessarily indicate a poor prognosis as long as adequate diagnosis and management are performed.

Case presentation: We report a case of a Moroccan female, aged 46 years old, who was diagnosed with both a breast cancer metachronous and rectal adenocarcinoma within an eleven years period.

The literature on multiple primary malignancies in a single patient is reviewed and the data are summarized.

Conclusion: From this rare case report and a review of the literature, it would appear that individuals who developed one malignancy might be at greater risk of developing a second.

The occurrence of multiple primary malignant neoplasms in our case may be explained by microsatellite instability, and increased surveillance. But there is no genetic predisposition.
\end{abstract}

Keywords: Multiple primary carcinomas; Breast; Rectal cancer

\section{Abbreviations}

ER: Estrogen Receptor; LFS: Li-Fraumeni Syndrome; MPMN: Multiple Primary Malignant Neoplasms; MSI: Microsatellite Instability; PR: Progesteron Receptor; RMN: Nuclear Magnetic Resonance

\section{Introduction}

As a result of improvements in diagnostic and therapeutic modalities in the field of oncology the number of patients who survive increase and the risk of developing a second tumour become important. Although the occurrence of multiple primary malignant neoplasms (MPMN) still a rare phenomenon.

Many theories have been proposed to explain MPMNs but none have been proven [1]. Studies are needed to define the magnitude of the problem and determine the predisposing factors. Additionally, multidisciplinary treatment and conservative strategies are important to improve quality of life and survival in these patients [1].

\section{Case Presentation}

A 46-year-old, non-smoking Moroccan female was diagnosed in 2002 with breast carcinoma, mammography detected a left breast cancer as an heterogeneous solid mass with irregular margins. A preoperative evaluation for distant metastases yielded negative results. A radical left mastectomy and axillary lymph-node dissection was performed, the histological type was a ductal invasive carcinoma and the stage was pT1N3M0 (Estrogen receptor negative, progesterone receptor positive, HER2/neu negative). She received adjuvant chemotherapy based on anthracyclines (6 cycles of doxorubicine 60 $\mathrm{mg} / \mathrm{m}^{2}$, and endoxan $600 \mathrm{mg} / \mathrm{m}^{2}$ ) and local standard radiotherapy (50 Gy with booster dose) just to the breast, with a small volume of underlying lung included in the high-dose volume. She then was placed on tamoxifen, she received 5 years. During the follow up, eleven years later, the patient presented with a history of diarrhea and bloody stool. A total colonoscopy demonstrated adenocarcinoma of the rectum at $5 \mathrm{~cm}$ from the anal verge. Pelvic Nuclear Magnetic resonance (NMR) showed a mass of the rectum at $3 \mathrm{~cm}$ from the anal verge with extent to the right sphincter. The extent of disease was evaluated by systemic physical examination and chest and abdominopelvic computed tomography which was negative.

After discussion, our mutidisciplinary team considered that this patient should have Preoperative chemoradiotherapy and surgery. 
Citation: Akasbi Y, Arifi S, Najib R, Hammas N, Amarti A, et al. (2015) An Unusual Case of Multiple Primary Carcinomas: Breast Cancer and Rectal Adenocarcinoma in a Single Patient: Report of a Case and Review of the Literature. Arch Surg Oncol 1: 107. doi: $10.4172 / 2471-2671.1000107$

Page 2 of 3

Preoperative radiation of a total dose of $50 \mathrm{~Gy}$ and concomitant chemotherapy with oral capecitabine was delivered. Intersphincteric resection colo-anal anastomosis with protectomy ileostomy and pelvic lymph nodes dissection was performed with clear resection margins. Histologically, the residual adenocarcinoma was ypT2N0M0 (Figure 1 and 2). The patient had a 2 years disease free survival.

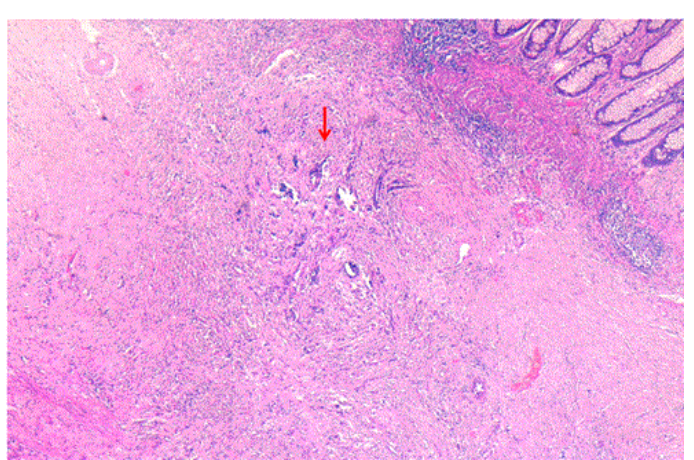

Figure 1: Carcinomatous glands infiltrating the muscularis (red arrow). Note the presence of the colon mucosa in the surface (top right) (H\&E staining). x 50).

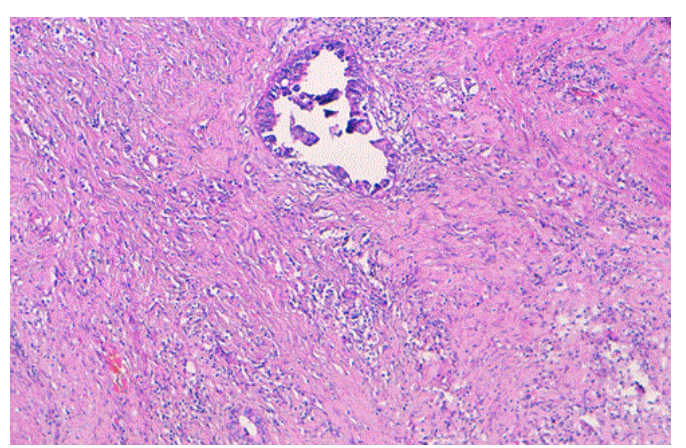

Figure 2: Represent the residual rectal adenocarcinoma, the Gland tumor was in the fibrous area corresponding to a therapeutic response (H\&E staining).x100.

No predisposing factor or a family history was found that might have contributed to the development of these malignancies.

\section{Discussion}

The report is of a patient who developed two histologically distinct malignancies, metachronous primary breast carcinoma, and a rectal adenocarcinoma, where the time between malignancies was more than 2 years.

Multiple primary cancers are defined as two or more cancers, with no subordinate relationship occurring either simultaneously or not in the same patient. No consensus is present on synchronous and metachronous tumor definition, the current trend is as follows: synchronous tumors are defined as second primary tumors occurring within 6 months of the diagnosis of the first primary tumor, whereas metachronous tumors were defined as those that were diagnosed after an interval of $>6$ months [1].
Most multiple primary cancers are double primary cancers, and the incidence decreases as the number of concomitant cancers increases. The incidence of multiple primary cancers is 2 to $6.3 \%$ of all cancers [1], while triple cancers occur in $0.5 \%$ of patients, and the incidence of quadruple primary cancers has been reported as $0.1 \%[2,3]$.

Multiple primary malignant neoplasms can occur at any age. However, from the reviewed series [2-5], the patients with MPMN tend to be older than those with a single primary malignant neoplasm. The mechanisms explaining the association of cancer and aging include:

- Time length of carcinogenesis; the longer a person lives the more likely it is that carcinogenesis will be completed and cancer will develop.

- Molecular changes of age; older tissues are susceptible to environmental carcinogenesis and undergo molecular changes similar to carcinogenesis.

- Changes in the environment; aging is associated with molecular changes in DNA signaling and body environment that may favor the development of cancer [3].

Women with a first primary breast cancer had a $25 \%$ increase in the risk of developing a new primary non breast cancer in comparison with women without cancer [6]. With the most consistent findings for cancer of the endometrium, ovary, thyroid gland, and lung, as well as soft tissue sarcomas and leukaemia, excesses of melanoma, stomach and colon cancer have also been reported [6].

We have to mention that the malignant tumors appeared after the primary cancer were not considered as rare individual observations, but as number of cases already taken into account. In our case, we dispose few case reports for the association of breast and rectal cancer [7-9].

The mechanism of multiple primary cancers is not fully known, but many hypotheses have been suggested, such as family history, immunologic and genetic defects, prolonged exposure to carcinogens, hormonal and metabolic disturbances, radiation and chemotherapy for the primary cancer, and field cancerization [10].

Iioka et al. [11] observed that patients with multiple primary cancers have a high frequency of microsatellite instability (MSI), arguing that MSI affects the pathogenesis of some multiple primary cancers. These may be explaining the occurrence of MPMN in our case. Unfortunately, we were not able to perform the MSI.

Primary multiple carcinoma was also described in Members of LiFraumeni syndrome families (LFS), germline mutations in the p53 tumor suppressor gene [12], Von Hippel Lindau syndrome and Multiple endocrine neoplasia Type 1.

Concerning our case, we did not fund the explanation of the association of breast and rectal cancer. The BRCA1 and BRCA2 genes confer increased susceptibility to breast and ovarian cancer ,But there is controversy regarding the incidence of colorectal cancer in BRCA1 and BRCA2 mutation carriers ;authors concluded from a follow-up study that the risk of colorectal cancer is increased in female carriers of BRCA1 mutations below the age of 50 years but not in women with BRCA2 mutations or in older women like our patient [13]; Also there is no role of chemotherapy (Anthracyclines and Cyclophosphamide ) in induction of colon cancer. 
Citation: Akasbi Y, Arifi S, Najib R, Hammas N, Amarti A, et al. (2015) An Unusual Case of Multiple Primary Carcinomas: Breast Cancer and Rectal Adenocarcinoma in a Single Patient: Report of a Case and Review of the Literature. Arch Surg Oncol 1: 107. doi: $10.4172 / 2471-2671.1000107$

Page 3 of 3

Recent studies have shown that the CHEK2 1100delC mutation identifies families with a hereditary breast and colorectal cancer phenotype. These findings may explain the association [14].

Authors found a relationship between the intake of omega-3 fatty acids and the risk of breast and colorectal but it doesn't mean they will represent a potential pathogenesis. There is still more work to be done to explain this association [15].

There are no established therapeutic rules for multiple primary cancers, but the type, progression, response to therapy, and patient's general health status should be considered. If each of cancers has the possibility for cure, radical therapy is indicated.

\section{Conclusion}

Multiple primary cancers are rarely reported, but it is believed that the incidence is rising. Prevention, early diagnosis, and treatment will become important and further studies are required.

The occurrence of multiple primary malignant neoplasms in our case may be explained by microsatellite instability, and increased surveillance. But there is no genetic predisposition.

\section{Consent}

Written informed consent was obtained from the patient for publication of this case report and accompanying images. A copy of the written consent is available for the Editor-in-Chief.

\section{Authors' Contributions}

Akabsi Y have drafting the work, and revising the manuscript. Arifi $S$ have revised the article critically for important intellectual content. Najib R contributed to the redaction of the manuscript. Hammas N have taken the figure. Amarti A contributed to the redaction of the manuscript. Mellas $\mathrm{N}$ have approved the version to be published.

All authors analyzed, interpreted and approved the final manuscript.

\section{References}

1. Kilciksiz S, Gokce Y, Baloglu A, Calli A, Kaynak C, et al. (2007) Characteristics of Synchronous- and Metachronous-Type Multiple Primary Neoplasms: A Study of Hospital-Based Cancer Registry in Turkey. Clin Genitourin Cancer 5: 438-445.
2. Noh SK, Yoon JY, Ryoo UN, Choi CH, Sung CO, et al. (2008) A case report of quadruple cancer in a single patient including the breast, rectum, ovary, and endometrium. J Gynecol Oncol 19: 265-269.

3. Hu NC, Hsieh SC, Chen TJ, Chang JY (2009) Multiple primary malignancies including colon, stomach, lung, breast, and liver cancer: a case report and literature review. Chin Med J (Engl) 122: 3091-3093.

4. Demandante CG, Troyer DA, Miles TP (2003) Multiple primary malignant neoplasms: case report and a comprehensive review of the literature. Am J Clin Oncol 26: 79-83.

5. Koutsopoulos AV, Dambaki KI, Datseris G, Giannikaki E, Froudarakis M, et al. (2005) A novel combination of multiple primary carcinomas: Urinary bladder transitional cell carcinoma, prostate adenocarcinoma and small cell lung carcinoma- report of a case and review of the literature. World J Surg Oncol 3: 51.

6. Mellemkjaer L, Friis S, Olsen JH, Scélo G, Hemminki K, et al. (2006) Risk of second cancer among women with breast cancer. Int J Cancer 118: 2285-2292.

7. Ewertz M, Mouridsen HT (1985) Second cancer following cancer of the female breast in Denmark, 1943-80. Natl Cancer Inst Monogr 68: 325-329.

8. Karayiannakis AJ, Kakolyris S, Kouklakis G, Chelis L, Bolanaki H, et al. (2011) Synchronous breast and rectal cancers in a man. Case Rep Oncol 4: 281-286.

9. Makis W, Ciarallo A, Lisbona R (2011) Three synchronous primary malignancies detected by F-18 FDG PET/CT: breast, rectal, and urothelial bladder carcinomas. Clin Nucl Med 36: 791-794.

10. Sorkin VM (2001) Breast cancer in women with prior malignant tumors. Lik Sprava : 95-98.

11. Iioka $\mathrm{Y}$, Tsuchida A, Okubo $\mathrm{K}$, Ogiso M, Ichimiya H, et al. (2000) Metachronous triple cancers of the sigmoid colon, stomach, and esophagus: report of a case. Surg Today 30: 368-371.

12. Hisada M, Garber JE, Fung CY, Fraumeni JF Jr, Li FP (1998) Multiple primary cancers in families with Li-Fraumeni syndrome. J Natl Cancer Inst 90: 606-611.

13. Phelan CM, Iqbal J, Lynch HT, Lubinski J, Gronwald J, et al. (2013) Hereditary Breast Cancer Study Group Incidence of colorectal cancer in BRCA1 and BRCA2 mutation carriers: results from a follow-up study. $\mathrm{Br}$ J Cancer 110: 530-534.

14. Meijers-Heijboer H, Wijnen J, Vasen H, Wasielewski M, Wagner A, et al. (2003) The CHEK2 1100delC mutation identifies families with a hereditary breast and colorectal cancer phenotype. Am J Hum Genet 72: 1308-1314.

15. Bartsch H, Nair J, Owen RW (1999) Dietary polyunsaturated fatty acids and cancers of the breast and colorectum: emerging evidence for their role as risk modifiers. Carcinogenesis 20: 2209-2218. 